\title{
Existence results for a class of Kirchhoff type systems with Caffarelli-Kohn-Nirenberg exponents
}

\author{
G.A. Afrouzi*, H. Zahmatkesh and S. Shakeri
}

\begin{abstract}
This paper is concerned with the existence of positive solutions for a class of infinite semipositone kirchhoff type systems with singular weights. Our aim is to establish the existence of positive solution for $\lambda$ large enough. The arguments rely on the method of sub-and supersolutions.
\end{abstract}

\section{Introduction}

In this article, we are interested in the existence of positive solutions for the following Kirchhoff type system

$$
\left\{\begin{array}{l}
-M_{1}\left(\int_{\Omega}|x|^{-a p}|\nabla u|^{p} d x\right) \operatorname{div}\left(|x|^{-a p}|\nabla u|^{p-2} \nabla u\right)=\lambda|x|^{-(a+1) p+c_{1}}\left(f(v)-\frac{1}{u^{\alpha}}\right) \quad \text { in } \Omega \\
-M_{2}\left(\int_{\Omega}|x|^{-b q}|\nabla v|^{q} d x\right) \operatorname{div}\left(|x|^{-b q}|\nabla v|^{q-2} \nabla v\right)=\lambda|x|^{-(b+1) q+c_{2}}\left(g(u)-\frac{1}{v^{\beta}}\right) \quad \text { in } \Omega \\
u=v=0 \text { on } \partial \Omega
\end{array}\right.
$$

where $\Omega$ is a bounded smooth domain of $R^{N}$ with $0 \in \Omega, 1<p, q<N, 0 \leq$ $a, b<\frac{N-p}{p}, c_{1}, c_{2}>0, \alpha, \beta \in(0,1), \lambda$ is a parameter, $f, g:(0, \infty) \rightarrow(0, \infty)$ are $C^{2}$ nondecreasing functions and $M_{1}, M_{2}: \Omega \rightarrow R$ satisfy the following condition

(H1) $M_{i}: R_{0}^{+} \rightarrow R^{+}, i=1,2$, are two continuous and increasing functions and $0<m_{i} \leq M_{i}(t) \leq m_{i, \infty}$ for all $t \in R_{0}^{+}$, where $R_{0}^{+}:=[0,+\infty)$.

Key Words: Nonlocal problems; Singular weights; infinite semipositone systems; Sub and supersolutions method.

2010 Mathematics Subject Classification: Primary 35J55, Secondary 35J65.

Received: 17.08 .2014

Accepted: 29.10.2014 
System (1) is related to the stationary problem of a model introduced by Kirchhoff [11]. More precisely, Kirchhoff proposed a model given by the equation

$$
\rho \frac{\partial^{2} u}{\partial t^{2}}-\left(\frac{P_{0}}{h}+\frac{E}{2 L} \int_{0}^{L}\left|\frac{\partial u}{\partial x}\right|^{2} d x\right) \frac{\partial^{2} u}{\partial x^{2}}=0,
$$

where $\rho, P_{0}, h, E$ are all constants. This equation extends the classical D'Alembert wave equation. A distinguishing feature of equation (2) is that the equations a nonlocal coefficient $\frac{P_{0}}{h}+\frac{E}{2 L} \int_{0}^{L}\left|\frac{\partial u}{\partial x}\right|^{2} d x$ which depends on the average $\frac{1}{2 L} \int_{0}^{L}\left|\frac{\partial u}{\partial x}\right|^{2} d x$; hence the equation is no longer a pointwise identity. Nonlocal problems can be used for modeling, for example, physical and biological systems for which $u$ describes a process which depends on the average of itself, such as the population density.

On the other hand, elliptic problems involving more general operator, such as the degenerate quasilinear elliptic operator given by $-\operatorname{div}\left(|x|^{-a p}|\nabla u|^{p-2} \nabla u\right)$, were motivated by the following Caffarelli, Kohn and Nirenberg's inequality (see $[4,19])$. The study of this type of problems motivated by its various applications, for example, in fluid mechanics, in newtonian fluids, in flow through porous media and in glaciology (see $[3,7]$ ). So, the study of singular elliptic problems has more practical meanings. We refer to $[9,16]$ for additional results on elliptic problems. Let $F(h, k)=f(k)-\frac{1}{h^{\alpha}}$, and $G(h, k)=g(h)-\frac{1}{k^{\beta}}$. Then $\lim _{(h, k) \rightarrow(0,0)} F(h, k)=-\infty=\lim _{(h, k) \rightarrow(0,0)} G(h, k)$ and hence we refer to (1.1) as an infinite semipositone problem. For regular case, that is, when $M_{1}(t)=M_{2}(t) \equiv 1, a=b=0, c_{1}=p$ and $c_{2}=q$ the quasilinear elliptic equation has been studied by several authors (see $[12,13])$. For the single equation case when $M_{1}(t)=M_{2}(t) \equiv 1, a=0, c_{1}=p=2$, see [17]. In [12], the authors extended the study of [17], to the corresponding systems, including p-Laplacian. Here we focus on further extending the study in [2, 15] for infinite semipositone Kirchhoff type systems involving singularity. Our approach is based on the sub- and super-solution method, see [1, 2, 6, 8, 10, 15]. The concepts of sub- and super-solution were introduced by Nagumo [20] in 1937 who proved, using also the shooting method, the existence of at least one solution for a class of nonlinear Sturm-Liouville problems. In fact, the premises of the sub- and super-solution method can be traced back to Picard. He applied, in the early 1880s, the method of successive approximations to argue the existence of solutions for nonlinear elliptic equations that are suitable perturbations of uniquely solvable linear problems. This is the starting point of the use of sub- and super-solutions in connection with monotone methods. Picard's techniques were applied later by Poincaré [21] in connection with problems arising in astrophysics.

We make the following assumptions: 
$(H 2) f, g:(0, \infty) \rightarrow(0, \infty)$ are $C^{2}$ nondecreasing functions $, f(0), g(0)>0$.

(H3) $\lim _{t \rightarrow \infty} g(t)=\infty$ and for all $M>0$

$$
\lim _{t \rightarrow \infty} \frac{f\left(M[g(t)]^{\frac{1}{q-1}}\right)}{t^{p-1}}=0 .
$$

Our main result is given by the following theorem.

Theorem 1.1. Under the conditions (H1)-(H3), there exists a positive constant $\lambda_{*}$ such that system (1) has a positive solution when $\lambda \geq \lambda_{*}$.

\section{Preliminary results}

In this paper, we denote $W_{0}^{1, p}\left(\Omega,|x|^{-a p}\right)$, the completion of $C_{0}^{\infty}(\Omega)$, with respect to the norm

$$
\|u\|_{p}=\left(\int_{\Omega}|x|^{-a p}|\nabla u|^{p} d x\right)^{\frac{1}{p}} .
$$

In order to precisely state our main result we first consider the following eigenvalue problem for the $p$-Laplace operator $-\Delta_{p} u$, see $[14,18]$ :

$$
\left\{\begin{array}{l}
-\operatorname{div}\left(|x|^{-a r}|\nabla u|^{r-2} \nabla u\right)=\lambda|x|^{-(a+1) r+c}|u|^{r-2} u \quad \text { in } \Omega, \\
u=0 \quad \text { on } x \in \partial \Omega .
\end{array}\right.
$$

Let $\phi_{1, r} \in C^{1}(\bar{\Omega})$ be the eigenfunction corresponding to the first eigenvalue $\lambda_{1, r}$ of (3) such that $\phi_{1, r}>0$ in $\Omega$ and $\left\|\phi_{1, r}\right\|_{\infty}=1$. It can be shown that $\frac{\partial \phi_{1, r}}{\partial \eta}<0$ on $\partial \Omega$ and hence, depending on $\Omega$, there exist positive constants $\epsilon, \delta, \sigma$ such that

$$
\left\{\begin{array}{l}
|x|^{-a r}\left|\nabla \phi_{1, r}\right|^{r}-\lambda_{1, r}|x|^{-(a+1) r+c} \phi_{1, r}^{r} \geq \epsilon \quad \text { on } \bar{\Omega}_{\delta}, \\
\phi_{1, r} \geq \sigma \quad \text { on } x \in \Omega \backslash \bar{\Omega}_{\delta},
\end{array}\right.
$$

where $\bar{\Omega}_{\delta}:=\{x \in \Omega: d(x, \partial \Omega) \leq \delta\}$ and $r=p, q$.

We will also consider the unique solution $e \in W_{0}^{1, p}(\Omega)$ of the boundary value problem

$$
\left\{\begin{array}{l}
-\operatorname{div}\left(|x|^{-a p}\left|\nabla e_{p}\right|^{p-2} \nabla e_{p}\right)=|x|^{-(a+1) p+c_{1}} \text { in } \Omega \\
\operatorname{div}\left(|x|^{-b q}\left|\nabla e_{q}\right|^{p-2} \nabla e_{q}\right)=|x|^{-(b+1) q+c_{2}} \text { in } \Omega, \\
e_{p}=e_{q}=0 \text { on } x \in \partial \Omega
\end{array}\right.
$$

to discuss our result. It is known that $e_{r}>0$ in $\Omega$ and $\frac{\partial e_{r}}{\partial \eta}<0$ on $\partial \Omega$ for $r=p, q$, see $[14]$. 
We shall establish our existence result via the method of sub and supersolutions. A pair of nonnegative functions $\left(\psi_{1}, \psi_{2}\right),\left(z_{1}, z_{2}\right)$ are called a subsolution and a supersolution of system (1) if they satisfy $\left(\psi_{1}, \psi_{2}\right)=(0,0)=\left(z_{1}, z_{2}\right)$ on $\partial \Omega$ and

$$
\begin{array}{r}
M_{1}\left(\int_{\Omega}|x|^{-a p}\left|\nabla \psi_{1}\right|^{p} d x\right) \int_{\Omega}|x|^{-a p}\left|\nabla \psi_{1}\right|^{p-2} \nabla \psi_{1} \nabla w d x \\
\leq \lambda \int_{\Omega}|x|^{-(a+1) p+c_{1}}\left(f\left(\psi_{2}\right)-\frac{1}{{\psi_{1}}^{\alpha}}\right) w d x \\
M_{2}\left(\int_{\Omega}|x|^{-b q}\left|\nabla \psi_{2}\right|^{q} d x\right) \int_{\Omega}|x|^{-b q}\left|\nabla \psi_{2}\right|^{q-2} \nabla \psi_{2} \nabla w d x \\
\leq \lambda \int_{\Omega}|x|^{-(b+1) q+c_{2}}\left(g\left(\psi_{1}\right)-\frac{1}{\psi_{2}{ }^{\beta}}\right) w d x
\end{array}
$$

and

$$
\begin{array}{r}
M_{1}\left(\int_{\Omega}|x|^{-a p}\left|\nabla z_{1}\right|^{p} d x\right) \int_{\Omega}|x|^{-a p}\left|\nabla z_{1}\right|^{p-2} \nabla z_{1} \nabla w d x \\
\geq \lambda \int_{\Omega}|x|^{-(a+1) p+c_{1}}\left(f\left(z_{2}\right)-\frac{1}{z_{1}^{\alpha}}\right) w d x \\
M_{2}\left(\int_{\Omega}|x|^{-b q}\left|\nabla z_{2}\right|^{q} d x\right) \int_{\Omega}|x|^{-b q}\left|\nabla z_{2}\right|^{q-2} \nabla z_{2} \nabla w d x \\
\geq \lambda \int_{\Omega}|x|^{-(b+1) q+c_{2}}\left(g\left(z_{1}\right)-\frac{1}{z_{2} \beta}\right) w d x
\end{array}
$$

for all $w \in W=\left\{w \in C_{0}^{\infty}(\Omega): w \geq 0\right.$ for all $\left.x \in \Omega\right\}$.

A key role in our arguments will be played by the following auxiliary result. Its proof is similar to those presented in [6], the reader can consult further the papers[1, 2, 10].

Lemma 2.1. Assume that $M: R_{0}^{+} \rightarrow R^{+}$is continuous and increasing, and there exists $m_{0}>0$ such that $M(t) \geq m_{0}$ for all $t \in R_{0}^{+}$. If the functions $u, v \in W_{0}^{1, p}\left(\Omega,|x|^{-a p}\right)$ satisfy

$$
\begin{aligned}
& M\left(\int_{\Omega}|x|^{-a p}|\nabla u|^{p} d x\right) \int_{\Omega}|x|^{-a p}|\nabla u|^{p-2} \nabla u \cdot \nabla \varphi d x \\
\leq & M\left(\int_{\Omega}|x|^{-a p}|\nabla v|^{p} d x\right) \int_{\Omega}|x|^{-a p}|\nabla v|^{p-2} \nabla v \cdot \nabla \varphi d x
\end{aligned}
$$

for all $\varphi \in W_{0}^{1, p}\left(\Omega,|x|^{-a p}\right), \varphi \geq 0$, then $u \leq v$ in $\Omega$. 
From Lemma 2.1 we can establish the basic principle of the sub- and supersolutions method for nonlocal systems. Indeed, we consider the following nonlocal system

$$
\left\{\begin{array}{l}
-M_{1}\left(\int_{\Omega}|x|^{-a p}|\nabla u|^{p} d x\right) \operatorname{div}\left(|x|^{-a p}|\nabla u|^{p-2} \nabla u\right)=|x|^{-(a+1) p+c_{1}} h(x, u, v) \text { in } \Omega, \\
-M_{2}\left(\int_{\Omega}|x|^{-b q}|\nabla v|^{q} d x\right) \operatorname{div}\left(|x|^{-b q}|\nabla v|^{p-2} \nabla v\right)=|x|^{-(b+1) q+c_{2}} k(x, u, v) \text { in } \Omega, \\
u=v=0 \text { on } x \in \partial \Omega,
\end{array}\right.
$$

where $\Omega$ is a bounded smooth domain of $R^{N}$ and $h, k: \bar{\Omega} \times R \times R \rightarrow R$ satisfy the following conditions

(HK1) $h(x, s, t)$ and $k(x, s, t)$ are Carathéodory functions and they are bounded if $s, t$ belong to bounded sets.

(KH2) There exists a function $g: R \rightarrow R$ being continuous, nondecreasing, with $g(0)=0,0 \leq g(s) \leq C\left(1+|s|^{\min \{p, q\}-1}\right)$ for some $C>0$, and applications $s \mapsto h(x, s, t)+g(s)$ and $t \mapsto k(x, s, t)+g(t)$ are nondecreasing, for a.e. $x \in \Omega$.

If $u, v \in L^{\infty}(\Omega)$, with $u(x) \leq v(x)$ for a.e. $x \in \Omega$, we denote by $[u, v]$ the set $\left\{w \in L^{\infty}(\Omega): u(x) \leq w(x) \leq v(x)\right.$ for a.e. $\left.x \in \Omega\right\}$. Using Lemma 2.1 and the method as in the proof of Theorem 2.4 of [14] (see also Section 4 of [5]), we can establish a version of the abstract lower and upper-solution method for our class of the operators as follows.

Proposition 2.2. Let $M_{1}, M_{2}: R_{0}^{+} \rightarrow R^{+}$be two functions satisfying the condition (H1). Assume that the functions $h, k$ satisfy the conditions (HK1) and (HK2). Assume that $(\underline{u}, \underline{v}),(\bar{u}, \bar{v})$, are respectively, a weak subsolution and a weak supersolution of system (7) with $\underline{u}(x) \leq \bar{u}(x)$ and $\underline{v}(x) \leq \bar{v}(x)$ for a.e. $x \in \Omega$. Then there exists a minimal $\left(u_{*}, v_{*}\right)$ (and, respectively, a maximal $\left.\left(u^{*}, v^{*}\right)\right)$ weak solution for system (7) in the set $[\underline{u}, \bar{u}] \times[\underline{v}, \bar{v}]$. In particular, every weak solution $(u, v) \in[\underline{u}, \bar{u}] \times[\underline{v}, \bar{v}]$ of system (7) satisfies $u_{*}(x) \leq u(x) \leq u^{*}(x)$ and $v_{*}(x) \leq v(x) \leq v^{*}(x)$ for a.e. $x \in \Omega$.

\section{Proof of the main result}

Proof. Choose $\eta>0$ such that $\eta \leq \min \left\{|x|^{-(a+1) p+c_{1}},|x|^{-(b+1) q+c_{2}}\right\}$ in $\bar{\Omega}_{\delta}$. For fixed $r_{1} \in\left(\frac{1}{p-1+\alpha}, \frac{1}{p-1}\right)$ and $r_{2} \in\left(\frac{1}{q-1+\beta}, \frac{1}{q-1}\right)$, we shall verify that

$$
\left(\psi_{1, \lambda}, \psi_{2, \lambda}\right)=\left(\lambda^{r_{1}} \eta^{\frac{1}{p-1}}\left(\frac{p-1+\alpha}{p}\right) \phi_{1, p}^{\frac{p}{p-1+\alpha}}, \lambda^{r_{2}} \eta^{\frac{1}{q-1}}\left(\frac{q-1+\beta}{q}\right) \phi_{1, q}^{\frac{q}{q-1+\beta}}\right),
$$

is a subsolution of (1). Let $w \in W$, Then a calculation shows that

$$
\nabla \psi_{1, \lambda}=\lambda^{r_{1}} \eta^{\frac{1}{p-1}} \phi_{1, p}^{\frac{1-\alpha}{p-1+\alpha}} \nabla \phi_{1, p}
$$




$$
\nabla \psi_{2, \lambda}=\lambda^{r_{2}} \eta^{\frac{1}{q-1}} \phi_{1, q}^{\frac{1-\beta}{q-1+\beta}} \nabla \phi_{1, q}
$$

and

$$
\begin{aligned}
& M_{1}\left(\int_{\Omega}|x|^{-a p}\left|\nabla \psi_{1, \lambda}\right|^{p} d x\right) \int_{\Omega}|x|^{-a p}\left|\nabla \psi_{1, \lambda}\right|^{p-2} \nabla \psi_{1, \lambda} \nabla w d x \\
& =\lambda^{r_{1}(p-1)} \eta M_{1}\left(\int_{\Omega}|x|^{-a p}\left|\nabla \psi_{1, \lambda}\right|^{p} d x\right) \int_{\Omega}|x|^{-a p} \phi_{1, p}^{\frac{(1-\alpha)(p-1)}{p-1+\alpha}}\left|\nabla \phi_{1, p}\right|^{p-2} \nabla \phi_{1, p} \nabla w d x \\
& =\lambda^{r_{1}(p-1)} \eta M_{1}\left(\int_{\Omega}|x|^{-a p}\left|\nabla \psi_{1, \lambda}\right|^{p} d x\right) \int_{\Omega}|x|^{-a p}\left|\nabla \phi_{1, p}\right|^{p-2} \nabla \phi_{1, p} \times \\
& \times\left[\nabla\left(\phi_{1, p}^{\frac{(1-\alpha)(p-1)}{p-1+\alpha}} w\right)-\left(\nabla \phi_{1, p}^{\frac{(1-\alpha)(p-1)}{p-1+\alpha}}\right) w\right] d x \\
& \leq \lambda^{r_{1}(p-1)} \eta m_{1, \infty} \int_{\Omega}\left[\lambda_{1, p}|x|^{-(a+1) p+c_{1}} \phi_{1, p}^{\frac{p(p-1)}{p-1+\alpha}}-|x|^{-a p} \frac{(1-\alpha)(p-1)}{p-1+\alpha} \frac{\left|\nabla \phi_{1, p}\right|^{p}}{\phi_{1, p}^{\frac{p p}{p-\alpha}}}\right] w d x .
\end{aligned}
$$

Similarly,

$$
\begin{aligned}
& M_{2}\left(\int_{\Omega}|x|^{-b q}\left|\nabla \psi_{2, \lambda}\right|^{q} d x\right) \int_{\Omega}|x|^{-b q}\left|\nabla \psi_{2, \lambda}\right|^{q-2} \nabla \psi_{2, \lambda} \nabla w d x \\
& \leq \lambda^{r_{2}(q-1)} \eta m_{2, \infty} \int_{\Omega}\left[\lambda_{1, q}|x|^{-(b+1) q+c_{2}} \phi_{1, q}^{\frac{q(q-1)}{p-1+\beta}}-|x|^{-b q} \frac{(1-\beta)(q-1)}{q-1+\beta} \frac{\left|\nabla \phi_{1, q}\right|^{q}}{\phi_{1, q}^{\frac{\beta q}{q-1+\beta}}}\right] w d x .
\end{aligned}
$$

First we consider the case when $x \in \bar{\Omega}_{\delta}$. We have $|x|^{-a p}\left|\nabla \psi_{1, p}\right|^{p} \geq \epsilon$ and $|x|^{-b q}\left|\nabla \psi_{1, q}\right|^{q} \geq \epsilon$ on $\bar{\Omega}_{\delta}$. Since $r_{1}(p-1) \geq 1-\alpha r_{1}$ and $r_{2}(q-1) \geq 1-\beta r_{2}$, we can find $\hat{\lambda}>0$ such that

$$
\begin{aligned}
& -\lambda^{r_{1}(p-1)} \eta m_{1, \infty}|x|^{-a p} \frac{(1-\alpha)(p-1)}{p-1+\alpha} \frac{\left|\nabla \phi_{1, p}\right|^{p}}{\phi_{1, p}^{\frac{\alpha p}{p-1+\alpha}}} \\
& \leq \lambda|x|^{-(a+1) p+c_{1}}\left(-\frac{1}{\left[\lambda^{r_{1}} \eta^{\frac{1}{p-1}}\left(\frac{p-1+\alpha}{p}\right) \phi_{1, p}^{\frac{p}{p-\alpha}}\right]^{\alpha}}\right),
\end{aligned}
$$

and

$$
\begin{aligned}
& -\lambda^{r_{2}(q-1)} \eta m_{2, \infty}|x|^{-b q} \frac{(1-\beta)(q-1)}{q-1+\beta} \frac{\left|\nabla \phi_{1, q}\right|^{q}}{\phi_{1, q}^{\frac{\beta q}{q-1+\beta}}} \\
& \leq \lambda|x|^{-(b+1) p+c_{2}}\left(-\frac{1}{\left[\lambda^{r_{2}} \eta^{\frac{1}{q-1}}\left(\frac{q-1+\beta}{q}\right) \phi_{1, q}^{\frac{q}{q-1+\beta}}\right]^{\beta}}\right),
\end{aligned}
$$


for all $x \in \bar{\Omega}_{\delta}$ and for all $\lambda \geq \hat{\lambda}$. Also since $r_{1}(p-1)<1$ and $r_{2}(q-1)<1$, we can choose $\check{\lambda}>0$ so that

$$
\begin{aligned}
\lambda^{r_{1}(p-1)} \eta m_{1, \infty} \lambda_{1, p}|x|^{-(a+1) p+c_{1}} \phi_{1, p}^{\frac{p(p-1)}{p-1+\alpha}} & \leq \lambda|x|^{-(a+1) p+c_{1}} f(0) \\
& \leq \lambda|x|^{-(a+1) p+c_{1}} f\left(\lambda^{r_{2}} \eta^{\frac{1}{q-1}}\left(\frac{q-1+\beta}{q}\right) \phi_{1, q}^{\frac{q}{q-1+\beta}}\right),
\end{aligned}
$$

and

$$
\begin{aligned}
\lambda^{r_{2}(q-1)} \eta m_{2, \infty} \lambda_{1, q}|x|^{-(b+1) q+c_{2}} \phi_{1, q}^{\frac{q(q-1)}{q-1+\beta}} & \leq \lambda|x|^{-(b+1) q+c_{2}} g(0) \\
& \leq \lambda|x|^{-(b+1) q+c_{2}} g\left(\lambda^{r_{1}} \eta^{\frac{1}{p-1}}\left(\frac{p-1+\alpha}{p}\right) \phi_{1, p}^{\frac{p}{p-1+\alpha}}\right),
\end{aligned}
$$

for all $x \in \bar{\Omega}_{\delta}$ and for all $\lambda \geq \check{\lambda}$.

Let $\lambda_{0}=\max \{\hat{\lambda}, \check{\lambda}\}$. Hence, for all $x \in \bar{\Omega}_{\delta}$ and for all $\lambda \geq \lambda_{0}$,

$$
\begin{aligned}
& M_{1}\left(\int_{\Omega}|x|^{-a p}\left|\nabla \psi_{1, \lambda}\right|^{p} d x\right) \int_{\bar{\Omega}_{\delta}}|x|^{-a p}\left|\nabla \psi_{1, \lambda}\right|^{p-2} \nabla \psi_{1, \lambda} \nabla w d x \\
& \leq \lambda \int_{\bar{\Omega}_{\delta}}|x|^{-(a+1) p+c_{1}} \\
& \times\left[f\left(\lambda^{r_{2}} \eta^{\frac{1}{q-1}}\left(\frac{q-1+\beta}{q}\right) \phi_{1, q}^{\frac{q}{q-1+\beta}}\right)-\frac{1}{\left(\lambda^{r_{1}} \eta^{\frac{1}{p-1}}\left(\frac{p-1+\alpha}{q}\right) \phi_{1, p}^{\frac{p}{p-1+\alpha}}\right)^{\alpha}}\right] w d x \\
& =\lambda \int_{\bar{\Omega}_{\delta}}|x|^{-(a+1) p+c_{1}}\left(f\left(\psi_{2, \lambda}\right)-\frac{1}{\psi_{1, \lambda}^{\alpha}}\right) w d x,
\end{aligned}
$$

and similarly

$$
\begin{aligned}
& M_{2}\left(\int_{\Omega}|x|^{-b q}\left|\nabla \psi_{2, \lambda}\right|^{q} d x\right) \int_{\bar{\Omega}_{\delta}}|x|^{-b q}\left|\nabla \psi_{2, \lambda}\right|^{q-2} \nabla \psi_{2, \lambda} \nabla w d x \\
& \leq \lambda \int_{\bar{\Omega}_{\delta}}|x|^{-(b+1) q+c_{2}}\left(g\left(\psi_{1, \lambda}\right)-\frac{1}{\psi_{2, \lambda}^{\beta}}\right) w d x .
\end{aligned}
$$

On the other hand, on $\Omega \backslash \bar{\Omega}_{\delta}$, we have $\phi_{1, r} \geq \sigma$, for some $0<\sigma<1$, and for $r=p, q$. Since $r_{1}(p-1)<1$ and $r_{2}(q-1)<1$ we can find $\tilde{\lambda}>0$ such that

$$
\begin{aligned}
& \lambda^{r_{1}(p-1)} \eta m_{1, \infty} \lambda_{1, p}|x|^{-(a+1) p+c_{1}} \phi_{1, p}^{\frac{p(p-1)}{p-1+\alpha}} \\
& \leq \lambda|x|^{-(a+1) p+c_{1}}\left[f\left(\lambda^{r_{2}} \eta^{\frac{1}{q-1}}\left(\frac{q-1+\beta}{q}\right) \sigma_{q}^{\frac{q}{q-1+\beta}}\right)-\frac{1}{\left(\lambda^{r_{1}} \eta^{\frac{1}{p-1}}\left(\frac{p-1+\alpha}{p}\right) \sigma_{p}^{p-1+\alpha}\right)^{\alpha}}\right],
\end{aligned}
$$


and

$\lambda^{r_{2}(q-1)} \eta m_{2, \infty} \lambda_{1, q}|x|^{-(b+1) p+c_{2}} \phi_{1, q}^{\frac{q(q-1)}{q-1+\beta}}$

$\leq \lambda|x|^{-(b+1) p+c_{2}}\left[g\left(\lambda^{r_{1}} \eta^{\frac{1}{p-1}}\left(\frac{p-1+\alpha}{p}\right) \sigma_{p}^{\frac{p}{p-1+\alpha}}\right)-\frac{1}{\left(\lambda^{r_{2}} \eta^{\frac{1}{q-1}}\left(\frac{q-1+\beta}{p}\right) \sigma_{q}^{\frac{q}{q-1+\beta}}\right)^{\beta}}\right]$,

for all $x \in \Omega \backslash \bar{\Omega}_{\delta}$ and for all $\lambda \geq \tilde{\lambda}$. Hence

$$
\begin{aligned}
& \lambda^{r_{1}(p-1)} \eta m_{1, \infty} \int_{\Omega \backslash \bar{\Omega}_{\delta}}\left[\lambda_{1, p}|x|^{-(a+1) p+c_{1}} \phi_{1, p}^{\frac{p(p-1)}{p-1+\alpha}}-|x|^{-a p} \frac{(1-\alpha)(p-1)}{p-1+\alpha} \frac{\left|\nabla \phi_{1, p}\right|^{p}}{\phi_{1, p}^{\frac{\alpha p}{p-1+\alpha}}}\right] w d x \\
& \leq \lambda^{r_{1}(p-1)} \eta m_{1, \infty} \int_{\Omega \backslash \bar{\Omega}_{\delta}} \lambda_{1, p}|x|^{-(a+1) p+c_{1}} \phi_{1, p}^{\frac{p(p-1)}{p-1+\alpha}} w d x \\
& \leq \lambda \int_{\Omega \backslash \bar{\Omega}_{\delta}} \lambda_{1, p}|x|^{-(a+1) p+c_{1}} \\
& \times\left[f\left(\lambda^{r_{2}} \eta^{\frac{1}{q-1}}\left(\frac{q-1+\beta}{q}\right) \sigma_{q}^{\frac{q}{q-1+\beta}}\right)-\frac{1}{\left(\lambda^{r_{1}} \eta^{\frac{1}{p-1}}\left(\frac{p-1+\alpha}{p}\right) \sigma_{p}^{\frac{p}{p-1+\alpha}}\right)^{\alpha}}\right] w d x \\
& \leq \lambda \int_{\Omega \backslash \bar{\Omega}_{\delta}}^{|x|^{-(a+1) p+c_{1}}\left(f\left(\psi_{2, \lambda}\right)-\frac{1}{\psi_{1, \lambda}^{\alpha}}\right) w d x .}
\end{aligned}
$$

and similarly

$$
\begin{aligned}
& \lambda^{r_{2}(q-1)} \eta m_{2, \infty} \int_{\Omega \backslash \bar{\Omega}_{\delta}}\left[\lambda_{1, q}|x|^{-(b+1) q+c_{2}} \phi_{1, q}^{\frac{q(q-1)}{q-1+\beta}}-|x|^{-b q} \frac{(1-\beta)(q-1)}{q-1+\beta} \frac{\left|\nabla \phi_{1, q}\right|^{q}}{\phi_{1, q}^{\frac{\beta q}{q-1+\beta}}}\right] w d x \\
& \leq \lambda \int_{\Omega \backslash \bar{\Omega}_{\delta}}|x|^{-(b+1) q+c_{2}}\left(g\left(\psi_{1, \lambda}\right)-\frac{1}{\psi_{2, \lambda}^{\beta}}\right) w d x .
\end{aligned}
$$

Let $\lambda_{*}=\max \left\{\lambda_{0}, \tilde{\lambda}\right\}$. Hence

$$
\begin{array}{r}
M_{1}\left(\int_{\Omega}|x|^{-a p}\left|\nabla \psi_{1, \lambda}\right|^{p} d x\right) \int_{\Omega}|x|^{-a p}\left|\nabla \psi_{1, \lambda}\right|^{p-2} \nabla \psi_{1, \lambda} \nabla w d x \\
\leq \int_{\Omega}|x|^{-(a+1) p+c_{1}}\left(f\left(\psi_{2, \lambda}\right)-\frac{1}{\psi_{1, \alpha}^{\alpha}}\right) w d x
\end{array}
$$


and

$$
\begin{array}{r}
M_{2}\left(\int_{\Omega}|x|^{-b q}\left|\nabla \psi_{2, \lambda}\right|^{q} d x\right) \int_{\Omega}|x|^{-b q}\left|\nabla \psi_{2, \lambda}\right|^{q-2} \nabla \psi_{2, \lambda} \nabla w d x \\
\leq \int_{\Omega}|x|^{-(b+1) p+c_{2}}\left(g\left(\psi_{1, \lambda}\right)-\frac{1}{\psi_{2, \lambda}^{\beta}}\right) w d x,
\end{array}
$$

i.e., $\left(\psi_{1, \lambda}, \psi_{2, \lambda}\right)$ is a subsolution of (1.1) for all $\lambda \geq \lambda_{*}$.

Now, we will prove there exists a $N$ large enough so that

$$
\left(z_{1}, z_{2}\right)=\left(N e_{p}(x),\left[\lambda g\left(N l_{p}\right)\right]^{\frac{1}{q-1}} e_{q}(x)\right),
$$

is a supersolution of (1.1), where $e_{r}$ is defined by (2.3) and $l_{r}=\left\|e_{r}\right\|_{\infty} ; r=p, q$. A calculation shows that:

$$
\begin{aligned}
& M_{1}\left(\int_{\Omega}|x|^{-a p}\left|\nabla z_{1}\right|^{p} d x\right) \int_{\Omega}|x|^{-a p}\left|\nabla z_{1}\right|^{p-2} \nabla z_{1} \nabla w d x \\
& \geq m_{0} N^{p-1} \int_{\Omega}|x|^{-a p}\left|\nabla e_{p}\right|^{p-2} \nabla e_{p} \nabla w d x \\
& =m_{0} N^{p-1} \int_{\Omega}|x|^{-(a+1) p+c_{1}} w d x .
\end{aligned}
$$

By monotonicity condition on $f$ and (H1)-(H3), we can choose $N$ large enough so that

$$
\begin{aligned}
m_{0} N^{p-1} & \geq \lambda f\left(\left[\lambda g\left(N l_{p}\right)\right]^{\frac{1}{q-1}} l_{q}\right) \\
& \geq \lambda f\left(\left[\lambda g\left(N l_{p}\right)\right]^{\frac{1}{q-1}} e_{q}(x)\right) \\
& =\lambda f\left(z_{2}\right) \\
& \geq \lambda\left(f\left(z_{2}\right)-\frac{1}{z_{1}^{\alpha}}\right) .
\end{aligned}
$$

Hence

$$
\begin{aligned}
& M_{1}\left(\int_{\Omega}|x|^{-a p}\left|\nabla z_{1}\right|^{p} d x\right) \int_{\Omega}|x|^{-a p}\left|\nabla z_{1}\right|^{p-2} \nabla z_{1} \nabla w d x \\
& \geq \lambda \int_{\Omega}|x|^{-(a+1) p+c_{1}}\left(f\left(z_{2}\right)-\frac{1}{z_{1}{ }^{\alpha}}\right) w d x .
\end{aligned}
$$


Next, we have

$$
\begin{aligned}
& M_{2}\left(\int_{\Omega}|x|^{-b q}\left|\nabla z_{2}\right|^{q} d x\right) \int_{\Omega}|x|^{-b q}\left|\nabla z_{2}\right|^{q-2} \nabla z_{2} \nabla w d x \\
& \geq m_{0} \lambda g\left(N l_{p}\right) \int_{\Omega}|x|^{-b q}\left|\nabla e_{q}\right|^{q-2} \nabla e_{q} \nabla w d x \\
& =m_{0} \lambda g\left(N l_{p}\right) \int_{\Omega}|x|^{-(b+1) q+c_{2}} w d x \\
& \geq \lambda \int_{\Omega}|x|^{-(b+1) q+c_{2}} g\left(N e_{p}(x)\right) w d x \\
& =\lambda \int_{\Omega}|x|^{-(b+1) q+c_{2}} g\left(z_{1}\right) w d x \\
& \geq \lambda \int_{\Omega}|x|^{-(b+1) q+c_{2}}\left(g\left(z_{1}\right)-\frac{1}{z_{2}^{\beta}}\right) w d x
\end{aligned}
$$

This relations show that $\left(z_{1}, z_{2}\right)$ is a supersolution of (1.1). Moreover, $z_{i} \geq \psi_{i, \lambda}$ for $N$ large, $i=1,2$. Thus, by Proposition 2.2 there exists a positive solution $(u, v)$ of $(1.1)$ such that $\left(\psi_{1, \lambda}, \psi_{2, \lambda}\right) \leq(u, v) \leq\left(z_{1}, z_{2}\right)$. This completes the proof of Theorem 1.1.

\section{References}

[1] G.A. Afrouzi, N.T. Chung and S. Shakeri, Existence of positive solutions for Kirchhoff type equations, Electron. J. Diff. Equ., Vol. 2013(180) (2013), 1-8.

[2] G.A. Afrouzi, N.T. Chung and S. Shakeri, Existence of positive solutions for Kirchhoff type systems with singular weights, to appear.

[3] C. Atkinson, K. El Kalli, Some boundary value problems for the Bingham model, J. Non-Newtonian Fluid Mech, (1992), No. 41, 339-363.

[4] L. Caffarelli, R. Kohn, L. Nirenberg, First order interpolation inequalities with weights, Compos. Math, (1984), No. 53, 259-275.

[5] A. Cañada, P. Drábek, J.L. Gámez, Existence of positive solutions for some problems with nonlinear diffusion, Trans. Amer. Math. Soc., 349 (1997), 4231-4249.

[6] N.T. Chung, An existence result for a class of Kirchhoff type systems via sub and supersolutions method, Appl. Math. Letters, (2013), to appear. 
[7] F. Cirstea, D. Motreanu, V. Radulescu, Weak solutions of quasilinear problems with nonlinear boundary condition, Nonlinear Anal, (2001), No. 43, 623-636.

[8] P. Drabek, J. Hernandez, Existence and uniqueness of positive solutions for some quasilinear elliptic problem, Nonlinear Anal, 2 (2001), No. 44, 189-204.

[9] F. Fang, S. Liu, Nontrivial solutions of superlinear p-Laplacian equations, J. Math. Anal. Appl., (2009), No. 351, 138-146.

[10] X. Han and G. Dai, On the sub-supersolution method for $p(x)$-Kirchhoff type equations, Journal of Inequalities and Applications, 2012 (2012): 283.

[11] G. Kirchhoff, Mechanik, Teubner, Leipzig, Germany, 1883.

[12] E.K. Lee, R. Shivaji, J. Ye, Classes of infinite semipositone $n \times n$ systems, Diff. Int. Eqs., (2011), No. 24, 361-370.

[13] E.K. Lee, R. Shivaji, J. Ye, Classes of infinite semipositone systems, Proc.Roy. Soc. Edinburgh 139A., (2009), No. 853-865.

[14] O.H. Miyagaki and R.S. Rodrigues, On positive solutions for a class of singular quasilinear elliptic systems, J. Math. Anal. Appl., 334(2) (2007), 818-833.

[15] S.H. Rasouli, On the existence of positive solutions for a class of infinite semipositone systems with singular weights, Thai Journal of Mathematics, Vol. 11 (2013), No. 1, 103-110.

[16] S.H. Rasouli, G.A. Afrouzi, The Nehari manifold for a class of concaveconvex elliptic systems involving the p-Laplacian and nonlinear boundary condition, Nonlinear Anal, (2010), No. 73, 3390-3401.

[17] M. Ramaswamy, R. Shivaji, J. Ye, Positive solutions for a class of infinite semipositone problems, Diff. Int. Eqs, 12 (2007), No. 20, 1423-1433.

[18] B. Xuan, The eigenvalue problem for a singular quasilinear elliptic equation, Electronic J. Diff. Equa., Vol. 2004 (2004), No. 16, 1-11.

[19] B. Xuan, The solvability of quasilinear Brezis-Nirenberg-type problems with singular weights, Nonlinear Anal, (2005), No. 62, 703-725.

[20] M. Nagumo, Über die Differentialgleichung $y^{\prime \prime}=f\left(x, y, y^{\prime}\right)$, Proceedings of the Physico-Mathematical Society of Japan 19 (1937), 861-866. 
[21] H. Poincaré, Les fonctions fuchsiennes et l'équation $\Delta u=e^{u}, J$. Math. Pures Appl. 4 (1898), 137-230.

G.A. Afrouzi,

Department of Mathematics,

Faculty of Mathematical Sciences,

University of Mazandaran, Babolsar, Iran.

Email: afrouzi@umz.ac.ir

H. Zahmatkesh,

Department of Mathematics,

Faculty of Mathematical Sciences,

University of Mazandaran, Babolsar, Iran.

Email: h.zahmatkesh@stu.umz.ac.ir

S. Shakeri,

Department of Mathematics,

Ayatollah Amoli Branch,

Islamic Azad University, amol, Iran.

Email: s.shakeri@iauamol.ac.ir 